\title{
Experimental and Numerical Study of Stagnant Zones in Pebble Bed
}

\author{
Xinlong Jia, ${ }^{1}$ Xingtuan Yang, ${ }^{1}$ Nan Gui, ${ }^{1}$ Yu Li, ${ }^{1}$ Jiyuan Tu, ${ }^{1,2}$ and Shengyao Jiang ${ }^{1}$ \\ ${ }^{1}$ Institute of Nuclear and New Energy Technology of Tsinghua University and the Key Laboratory of \\ Advanced Reactor Engineering and Safety, Ministry of Education, Beijing 100084, China \\ ${ }^{2}$ School of Aerospace, Mechanical \& Manufacturing Engineering, RMIT University, Melbourne, VIC 3083, Australia \\ Correspondence should be addressed to Shengyao Jiang; jiangshy2012@sina.com
}

Received 17 December 2013; Accepted 11 April 2014; Published 18 May 2014

Academic Editor: Shengqiang Li

Copyright (C) 2014 Xinlong Jia et al. This is an open access article distributed under the Creative Commons Attribution License, which permits unrestricted use, distribution, and reproduction in any medium, provided the original work is properly cited.

\begin{abstract}
The experimental method (side area method) and DEM simulation have been carried out to analyse the stagnant zone in the quasitwo-dimensional silos. The side area method is a phenomenological method by means of investigating the interface features of different areas composed of different coloured pebbles. Two methods have been discussed to define the stagnant zone. In particular, the area of the stagnant zone has been calculated with the mean-streamline method, and the tracking time of different marking pebbles has been investigated with the stagnant time method to explore the kinematics characteristics of the pebbles. The stagnant zone is crucial for the safety of the pebble-bed reactor, and the practical reactor core must avoid the existence of the stagnant zone. Furthermore, this paper also analyses the effects of bed configuration (the bed height, the base angle, and the friction coefficient) on stagnant zone with the two methods mentioned above. In detail, the bed height shows little impact on the stagnant zones when the bed height exceeds a certain limit, while the base angle has negative prominent correlation with the stagnant zone. The friction coefficient effect seems complicated and presents the great nonlinearity, which deserves to be deeply investigated.
\end{abstract}

\section{Introduction}

It is generally known that the high temperature gas-cooled nuclear reactor is regarded as one potential solution for generation IV advanced reactor [1]. The Institute of Nuclear and New Energy Technology (INET) at Tsinghua University has developed the HTR-PM [2], which has been authorized to be one of the National Special Grand Science-Technology Projects of China.

In addition, the mechanisms and characteristics of the pebble-bed reactor have received more attention. Some countries, such as South Africa [3] and the United States [4], develop their own test and demonstration reactors (PBMR and MPBR, resp.) and their prototype reactor, for example, the AVR early in Germany, and so forth [5].

The geometric and physical design of pebble-bed reactor core is based on the flow characteristics of the spherical fuel elements in the pebble bed. The pebble flow (or named granular flow) is an attractively simple and yet surprisingly complex area of research [6]. Fast, dilute flows are generally dominated by classical hydrodynamics (with inelastic collisions), while slow, dense flows seem full of challenge, because of many-body interactions and nonthermal fluctuations. Such flows are meaningful for not only the fundamental scientific research, but also the engineering applications [7]. For instance, the degree of mixing in very slow granular drainage $(<1$ pebble per min) determines the efficiency and safety of the new pebble-bed nuclear reactors [8].

Currently, the information about the dense pebble flow provided by experiments is limited, but important. Numerous experiments of the drainage flows in quasi-two-dimensional silos have been conducted. The motion information of the particles is tracked accurately at a transparent wall [9-13]. The technology, such as the magnetic resonance imaging [14], confocal microscopy[15], index matching with an interstitial fluid [16], and diffusing-wave spectroscopy [17], has been applied in particle tracking in some 3D granular materials and colloids. However, there are obvious distinctions between the experiment equipment and the practical pebble-bed reactor core. The porosity distribution of static packing of 

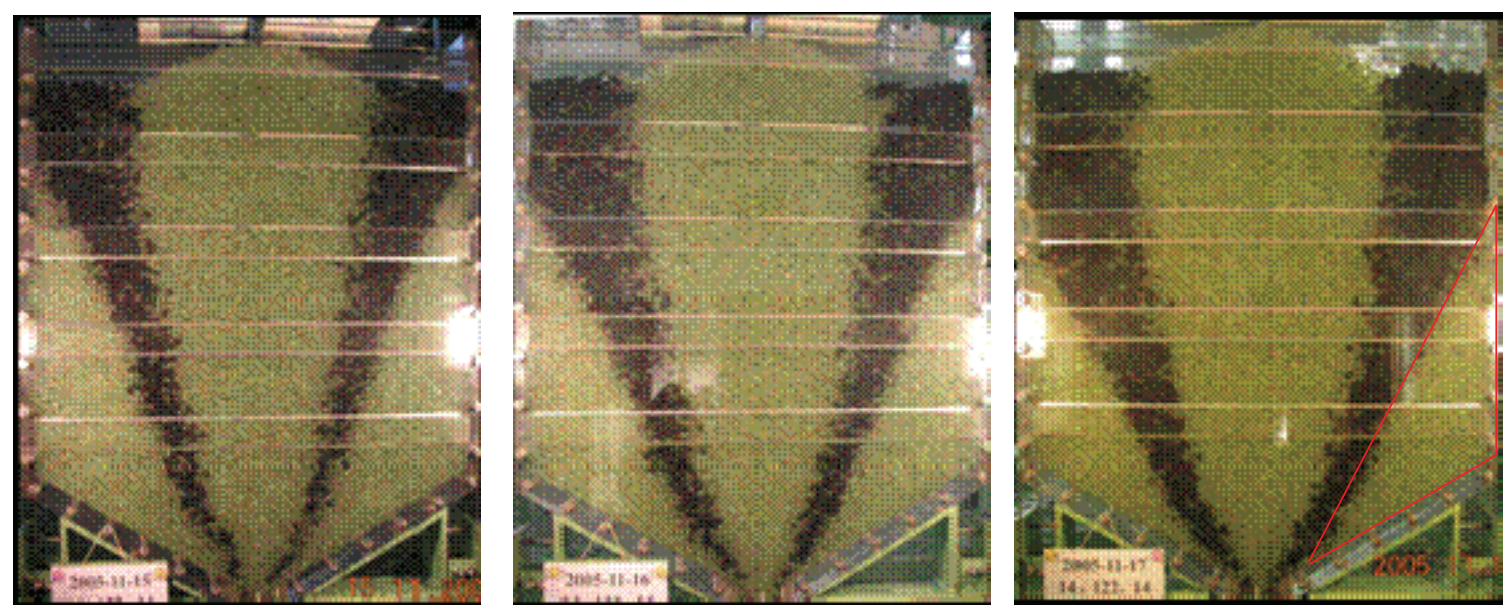

FIGURE 1: Sketch of the stagnant zone of different time with the side area method in experiment.

spheres [18] has drawn more attention in the experiment about the realistic geometries for PBR, because it affects helium gas flow through the core [19].

Moreover, the past 50 years witnessed the developing of the mathematical models of the pebble flow, including the continuum approach [20], void model [21], spot model [22], and discrete element method [23] (DEM). However, a generally accepted theoretical model has not been found yet. Among them, DEM with its qualitative accuracy might be the most applicable approach for dense granular flow investigation [24].

There are some features concerning the quasi-static dense pebble flow in the pebble-bed reactor. First of all, the pebbles are always undergoing the transition from static state to dynamic state, and then back to static state with small disturbance, which are only driven by the gravity. Additionally, this shows that the falling pebbles in the pebble packing swiftly reachieve their own equilibrium state with almost no collision effects [24], and the equilibrium state time is larger than the dynamic state time. As mentioned above, the quasi-static dense pebble flow in the pebble-bed reactor is quite different from the normal dilute flow, which needs to be explored.

Stagnant zone can be found in the low corners, or at the bottom of the cone angle of the experimental vessels near the wall under some conditions [24]. Figures 1 and 2 display the sectional sketch map of the stagnant zone marked with the red outline in the experimental vessel. A stagnant zone in the pebble bed probably occurs under the specific design of a reactor core. Pebbles in the stagnant region will move extremely slowly or even remain static. The fuel pebbles which stay in the stagnant zone and spend much more time than their design parameter may cause the severe irradiation and thermal damage with possible fission product escaping. So stagnant region is not allowed to exist in practical reactors, and the strategy to eliminate the stagnant zone should be discussed.

Investigation on parameter effects of pebble flow and the stagnant zone in the dense pebble flow is meaningful and significant to understand the physical mechanism of quasi-static dense flow. However, the study of the field of pebble flow of pebble-bed reactor is not sufficient by now, and only few literatures were published. For example, MIT's scientists have ever reported their research results under the operation mode of free drainage of pebbles [4, 25].

Motivated by this consideration, the experiment and the discrete element method (DEM) simulation will be carried out to investigate the stagnant zone forming in the lower corners of the vessel. More motion behaviors and characteristics of the dense pebble flow will be observed. Furthermore, we try some quantitative methods to calculate and analyze the stagnant zone in the dense pebble flow, and parameter effects of the stagnant zone will be discussed. All the work may help us have a more profound understanding of the characteristics of the dense pebble flow in the pebble-bed reactor.

\section{Experimental Setup and Numerical Method}

2.1. Experimental Setup. The experimental vessel is a $2 \mathrm{D}$ structure with smaller size, shown in Figure 1, which is designed to simulate a real pebble-bed reactor at the scale of $1: 5$. This experiment facility runs in a way of recirculation mode; that is to say, the pebbles discharged from the outlet will be carried back to the inlet tube.

The experiment facility has the dimensions $3000 * 3000 *$ $6000 \mathrm{~mm}$ in total length, width, and height, respectively [4]. The main three parts make up the whole experimental setup. Firstly, the vessel (Figure 1) is made up of the plexiglass, with the dimensions $800 * 1000(2000) * 120 \mathrm{~mm}$ in width, height (after being increased), and thickness, respectively. It is a quasi-two-dimensional setup. The bottom of the vessel has a steep hill of 30 degrees. The pebbles in the vessel are made up of glass, with the numbers 70000 and 150000 (after being increased). Moreover, there are three inlet tubes over the vessel, and the pebbles from the inlet tubes will be falling in the left, center, and right zones in the upper surface of the packing pebbles in the vessel. The discharge hole is in the bottom of the vessel, with the diameter of $120 \mathrm{~mm}$ and length of $200 \mathrm{~mm}$. 
2.2. Numerical Method. Discrete element method (DEM) is usually applied in the research about the discontinuous media. The calculated zones are covered by collections of unique "discrete elements". Every element has its own independent properties and motion parameters. One hypothesis of the DEM indicates that the contacting interactions of elements with each other are proportional to the relative displacement between them. The equations of motion of the elements will be established based on Newton's laws.

The following equations described the translational and rotational motions of element " $i$ " in a system at time $t$, determined by Newton's second law:

$$
\begin{gathered}
m_{i} \frac{d V_{i}}{d t}=\sum_{j=1}^{n} F_{j i}^{c}+F_{i}^{g}, \\
I_{i} \frac{d w_{i}}{d t}=\sum_{j=1}^{n}\left(r_{i j} \times F_{j i}^{c}\right)+M_{i}^{e} .
\end{gathered}
$$

In the equations above, $m_{i}, I_{i}, V_{i}$, and $w_{i}$ represent the mass, moment of inertia, and translational and rotational velocities of particle " $i$ "; $F_{i}^{g}$ is the gravitational force; $r_{i j}$ is the vector pointing from element " $i$ " to " $j$ ".

The contact force $F_{j i}^{c}$ includes two parts: normal contact force $F_{j i}^{c n}$ and tangential force $F_{j i}^{c s}$, and they are shown as follows:

$$
\begin{gathered}
F_{j i}^{c n}=-k_{n} \cdot \Delta u_{i j}^{n}+\beta_{n} \cdot V_{j i}^{n}, \\
F_{j i}^{c s}=-k_{s} \cdot \Delta u_{i j}^{s}+\beta_{n} \cdot V_{j i}^{s}, \\
\left|F_{j i}^{c s}\right|_{\max } \leq \mu\left|F_{j i}^{c n}\right|,
\end{gathered}
$$

where $k$ and $\beta$ represent the stiffness and damping coefficients, respectively, and $n$ and $s$ represent the normal and tangential directions, respectively. $\mu$ is the friction coefficient; $\Delta u_{i j}^{n}$ and $\Delta u_{i j}^{s}$ delegate the normal and tangential deformations; and $\Delta V_{i j}^{n}$ and $\Delta V_{i j}^{s}$ are the normal and tangential relative velocities of two contacted particles.

The DEM simulation is carried out in a quasi-twodimensional condition too, according to the experimental setup for comparative study. Moreover, the interaction model in DEM is called the "spring-dashpot" model. It is suitable for present simulation since the particle material is plexiglass where the collision between the particles is considered as incomplete elastic because of the damping effect of the material.

\section{Results and Discussions}

\subsection{Observation of Stagnant Zone}

3.1.1. Experimental Observation. Side area method (Figure 1) can be designed to investigate the stagnant zone in the lower corners of the vessel, which focuses on the characteristics of the interface between different color pebbles.

The experiment begins with the random packing by prefilling the colorless pebbles in the vessel. Then, colorless pebbles were falling from the middle inlet tube, and black pebbles were falling from both sides of inlet tubes, with the ratio of $14: 122: 14$. At the same time, pebbles were flowing from the discharge hole with 150 pebbles per minute. The stagnant zone is most possible to occur in both corners of the vessel. The initially filled pebbles which still remained in the vessel are surrounded by the inserted black pebbles and seem more conspicuous, as shown in Figure 1.

As is illustrated in Figure 1, the black pebbles area and the sides and bases of the vessel enclose up the two stagnant zones, where the initially filled pebbles still remained in the vessel. After about $22 \mathrm{~h}$ of operation, the stagnant zone still remained with about 2.8 times of the total number recirculated. However, the pebbles in stagnant zone were not completely static. It is obvious that the size of the stagnant zone decreased with the recirculation continuing. Figure 1 shows the size of the stagnant zones after $22 \mathrm{~h}, 40 \mathrm{~h}$, and $60 \mathrm{~h}$, respectively, but finally the stagnant zone stayed with a stable size and did not change any more, which illustrates that the stagnant zone exists under the experimental conditions. This is apparently unacceptable in practical reactor core.

3.1.2. Simulation Observation. Similarly, the forming of stagnant zone has been carried out with the DEM simulation. The parameters of the simulation are the same with the above experiment. Firstly, green pebbles were prefilled to form the initial random packing state; then, green pebbles were falling from the middle inlet tube, and red pebbles were falling from both sides of inlet tubes, with the ratio of $14: 122: 14$.

Figure 2 shows the DEM simulation results. By comparing Figure 1 with Figure 2, it indicates that DEM simulation is remarkably in agreement with experiment results, where the friction coefficient of both particle-particle and particle-wall is 0.2 and cone angle is $30^{\circ}$.

3.2. Stagnant Zone Definition. The movement information, such as the mean-streamline and the mean stagnant time, of the marking pebbles in different radial position could reflect the statistical characteristics of the movement of the pebbles when there is plenty of statistical data. This paper shows two different methods to define the stagnant zone in order to analyze the effects of the bed configuration on the shape, size, and other mechanical mechanisms and characteristics of the stagnant zone.

The mean-streamline method to define the stagnant zone is mainly based on the section about the experimental observation of the stagnant zone with the side area method. As shown in Figure 1, the obvious interface of the experiment between the white pebbles and black pebbles will finally remain stable as time went on; that is to say, the size of the white pebble zone and the black pebble zone will not change any more. The scope of the small mixing zone (Figure 4) between the white and black pebble zones is limited; that is to say, the curved surface $S$ exists which is made up of the mean-streamlines of the mixing zone. If the time-average flux of the pebble flow through the interface between the different color pebbles is not equal to zero, then the smooth, stable interface will not exist. In summary, the curved surface $S$ and 


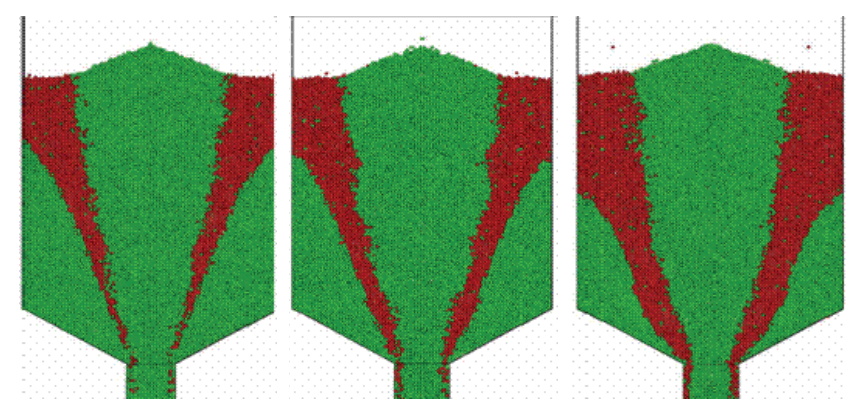

FIGURE 2: Sketch of the stagnant zone of different time with the side area method with DEM method.

the interface of the two zones coincide with each other, when the central zone and the side zone stay stable.

The mean stagnant time method to define the stagnant zone is mainly based on the marking pebble method. As shown in Figure 5, the stagnant time of the marking pebbles from the upper surface to the outlet hole in the vessel will be recorded, which will be meaningful for analyzing the pebble flow when the data is enough. This method can also get the information about the mean-streamline.

3.2.1. Mean-Streamline. In the experiment with $h=1 \mathrm{~m}$, the mean-streamlines of the pebbles after $t=60 \mathrm{~h}$ in the pebble bed are smooth and symmetrical. The base cone lacks the arc transition, which causes the existence of the very slow flow zone.

As easily inferred, when the pebbles fall through the cylinder vessel where the outlet tube or orifice has the same diameter of the main body, that is to say, when the pebbles are regarded as a whole body, they are only affected by the gravity and the friction with the wall. The streamlines of the pebbles are almost vertically downward, and the stagnant zone will disappear resulting from the changing of the vessel shape.

Considering the influence of the geometric factor, the size of stagnant zone can be defined as follows: the stagnant zone is the area in the corner of the cone-base section between mean-streamline in the side area method where pebble is nestling against the wall of the vessel at initial time and the outline of the wall of the vessel which is the green zone in Figure 3, when the region shape remains a dynamic equilibrium or time stationary. Then, the area of the stagnant zone $A_{s}$ can be calculated.

A mixing zone exists between the stagnant zone and the central zone illustrated in Figure 4 which makes the stagnant zone have a blurry boundary. The mixing zone is crucial and is required to be small enough in the real pebble-bed reactor. One way to minimize the mixing zone is to insert plates [24] in the separating surfaces between the central region and the side region at the top section of the vessel. In addition, the competency of the guide ring in a practical reactor needs more theoretical analysis and experimental verifications. The bad influence of the mixing zone on defining the size of the stagnant zone will be reduced when using the meanstreamline method from the statistical point of view.

To analyze the relationship of the size of the stagnant zone and the whole pebble bed, it is necessary to define the

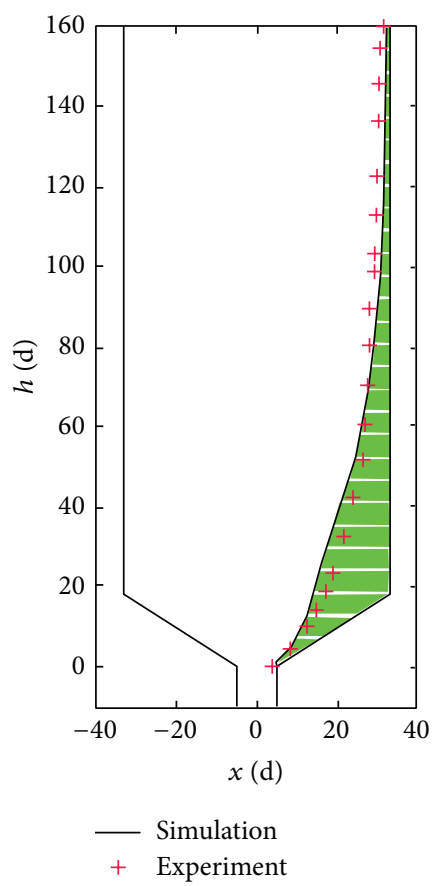

FIGURE 3: The mean-streamline method of the definition of stagnant zone $\left(f=0.2, A=30^{\circ}\right.$, and $\left.h=160 \mathrm{~d}\right)$.

stagnant zone area fraction $\alpha_{s}$ which is the ratio of the area of stagnant zone $A_{s}$ to the whole zone $A_{w}$ which is filled with the pebbles in the pebble bed.

3.2.2. Mean Stagnant Time. Figure 5 shows the distribution of the mean stagnant time of the pebbles in the experiment ( $h=1 \mathrm{~m}$ ). Because the stagnant time is related to the load and drainage rate of the pebbles, the critical stagnant time hardly takes effect about the stagnant zone definition actually. It is more reasonable to describe the characteristics of the pebble flow when the mean stagnant time is replaced by the drainage pebble rate. That is to say, paying more attention to the number of the drainage pebbles before the marking pebble flows out of the orifice is more meaningful. But the experimental data in this paper is received in the regular or fixed drainage pebble rate, so there is much comparability in the mean stagnant time of different pebbles in the experiment installation. 


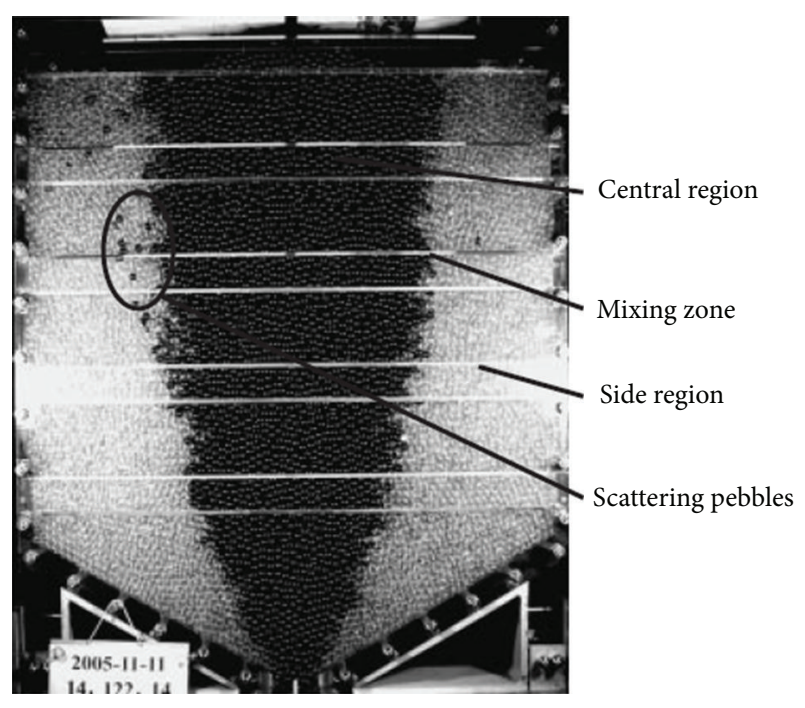

FIGURE 4: Establishment of the two-region arrangement in experiment without guide plates.

The stagnant zone can be defined as follows: the stagnant zone is the area where the data of the mean stagnant time of pebbles or the element balls in the pebble bed exceed the limit or design value. The pebbles in the stagnant zone are not critically static, although they cannot flow out of the orifice in a certain time.

The mean stagnant time method is diverse from the mean-streamline method in defining the stagnant zone in the following aspects. Firstly, the mean stagnant time method is usually effective when used to describe the change of the size of the stagnant zone dynamically. On the contrary, the mean-streamline method focuses on the stagnant zone when it gets its steady state. Meanwhile, the mean-streamlines are basically unchanged. Secondly, the mean-streamline method describes the stagnant zone in space, but the mean stagnant time method focuses on time. Thirdly, the data must be enough for the mean stagnant time method to show the meaningful statistical information, while it is not necessary for the mean-streamline method. The mean-streamline method has more advantages than the mean stagnant time method in attaining the pebble flow information in 3D experiments. But the results of the mean stagnant time seem more direct and visual and show more quantification. Also, in the nuclear reactor, the stagnant zone is the area where the burnup fraction of the fuel element can reach a certain value after which it goes through the reactor core one time in the pebble flow. This method is complicated because it is related to the thermal reactor physics and needs much more data, such as the velocity and the route of the movement of the pebbles.

In this paper, we do not use the burnup fraction method because it cannot give a precise definition of the stagnant zone. The size of the stagnant zone is changing with time and finally becomes fixed. In this paper, we mainly use the meanstreamline method and the mean stagnant time method.

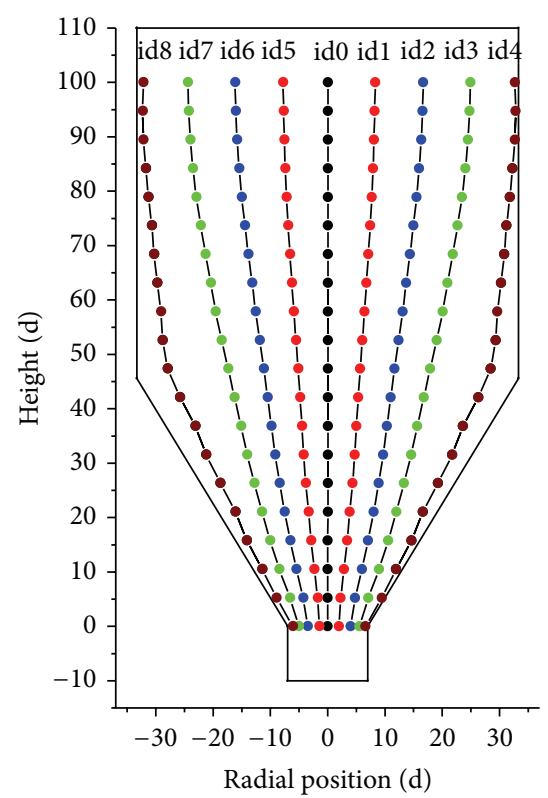

FIGURE 5: Distribution of the mean stagnant time and the streamline of the marking pebbles in the experiment $(h=75 \mathrm{~d})$.

3.3. Effects of Bed Configuration on Stagnant Zone. Investigation on parameter effects of pebble flow is meaningful and significant to understand the physical mechanism of quasi-static pebble flow and to explore how to minimize the stagnant zone.

In this section, we will provide some information of parameter effect on the pebble packing under recirculation operation mode, including different height of the experiment installation, different friction coefficient, and different base cone angle. In order to facilitate the comparison, only one parameter was altered in one case; the rest such as operation mode, rate of discharging, and ratio of loading were unchanged.

Geometric design plays the most important part in the pebble flow, including configuration of vessel, height, width, discharging hole diameter, pebble diameter, and base cone angle, on which some important dimensionless numbers have been derived. We only investigate the height; base cone angle influences the pebble flow under our experiment conditions here.

3.3.1. The Bed Height Effect. The simulation and experiment have been carried out to analyze the stagnant zone in the pebble-bed reactor. Figure 6 shows the results of DEM simulation on different bed heights from $h=75 \mathrm{~d}$ to $h=$ $160 \mathrm{~d}$ on the base cone angle $30^{\circ}$ under $f=0.2$. The ratio of loading pebbles was set to be $14: 122: 14$; discharging pebble was 150 pebbles per minute. Snapshots were taken under the side-inserting pebble manner at intervals to record the changing of the size of the stagnant zone. Only a part of them were presented here.

The DEM simulation of the changing process of the stagnant zone on different bed height demonstrates the following: the changing process of the size of the stagnant 

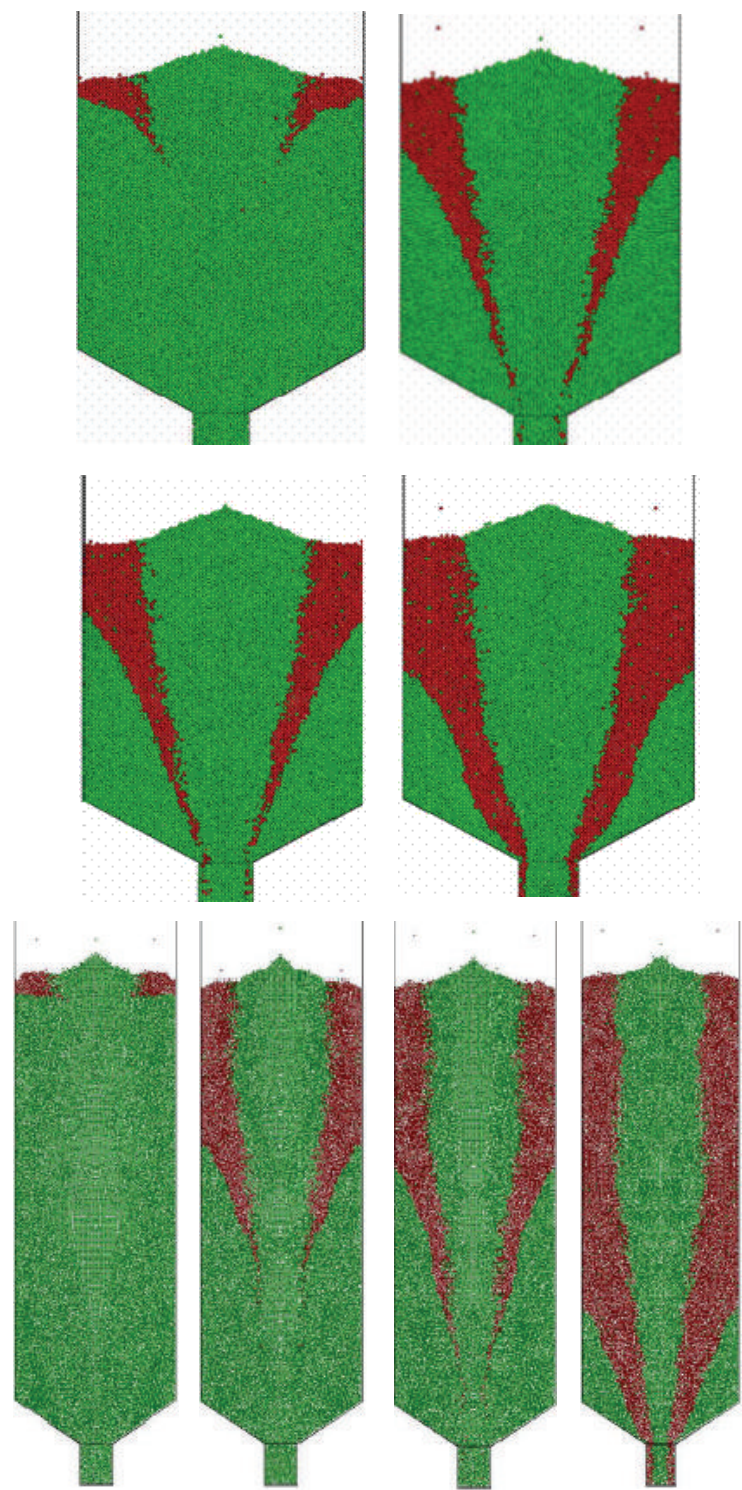

FIGURE 6: DEM simulation of stagnant zone on different bed height from $h=75 \mathrm{~d}$ to $h=160 \mathrm{~d}\left(f=0.2, A=30^{\circ}\right)$.

zone can be affected by the pebble-bed height. This change is related to the fact that more time will be needed for the pebbles to flow through the pebble bed with the height of 160 $\mathrm{d}$ under the same discharging rate. That is to say, pebbles with the same velocity require more flow time for transferring a longer distance. The changing of the height of the pebble bed almost has an impact on the pebbles in the lower corner of the pebble bed initially. Meanwhile, the lateral or radial diffusion of the pebbles tends to become more intensive with the more space and time provided on the condition of $h=160 \mathrm{~d}$.

Figures 5 and 7 show the final results about the distribution of the mean-streamlines of the marking pebbles from id0 to id 8 in the experiments with $h=75 \mathrm{~d}$ and $h=160 \mathrm{~d}$ on the base cone angle $30^{\circ}$ under $f=0.2$ with the same loading and discharging rate. The position of the pebbles from id0 to id8 presents a symmetrical distribution.

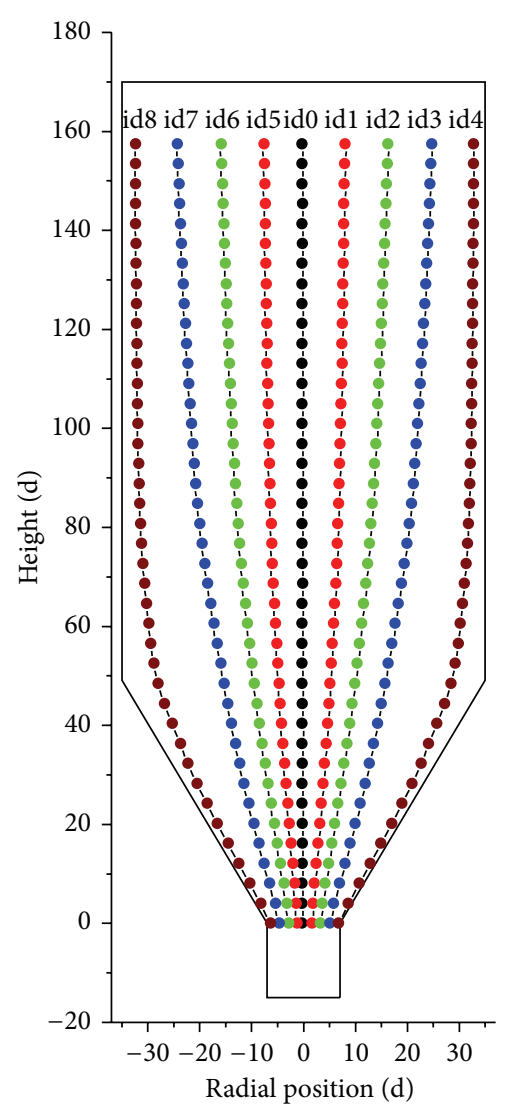

FIGURE 7: Distribution of the mean stagnant time and streamline of the marking pebbles in the experiment $(h=160 \mathrm{~d})$.

The experiment results of the mean-streamlines with different bed height denote the following.

The streamlines of the marking pebbles in the pebble bed are almost smooth and symmetrical. Pebbles in the lower corner of the pebble bed flow more slowly, and this zone may have a significant influence on the pebble flow which makes the streamline of id 4 unable to adapt to the shape of the wall. Then, a triangle zone exists between the streamline and the wall named the stagnant zone. The pebbles in the stagnant zone are almost static which squeeze the central pebbles intensively and cause the flow irregularity in the pebble bed; as a result, the bend of the streamline exists much earlier than that of the wall of the pebble bed. As shown in Figure 7, about experiment results of the distribution of the meanstreamlines of the experiment with $h=160 \mathrm{~d}$, the meanstreamlines in the upper zone of the pebble bed are almost vertical, which are different from those in the lower zone. From the perspective of the streamlines, the upper zone of the pebble bed may have a particularly small impact on the lower zone of the pebble bed. In other words, the streamlines in the lower zone of the experiment with $h=160 \mathrm{~d}$ may have an identical shape with those in the experiment with the height $h=75 \mathrm{~d}$.

As mentioned above, the streamlines of the upper zone of the pebble bed with height $h=160 \mathrm{~d}$ may have little influence on the size of the stagnant zone with the mean-streamline 


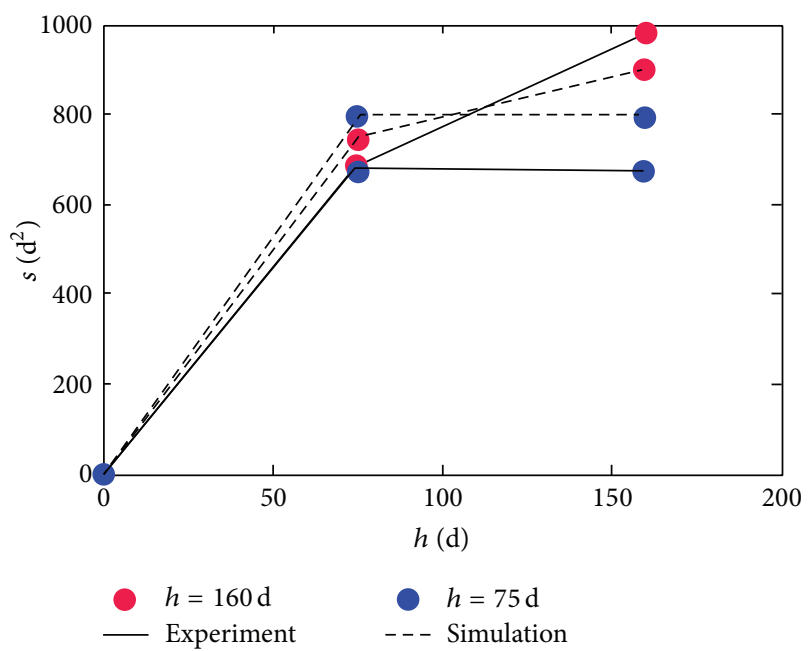

FIGURE 8: The relationship between the height of the pebble bed and the area of the stagnant zone about the experimental and simulation results.

definition method. To verify this consideration, the area of the stagnant zone $A_{s}$ on the core angle $30^{\circ}$ under $f=0.2$ has been calculated in the experiments with $h=75 \mathrm{~d}$ and $h=160 \mathrm{~d}$ with the data of the experiment and simulation given in Figure 8.

The relationship between the height of the pebble bed and the area of the stagnant zone in Figure 8 can be illustrated as follows.

(1) The $A_{s}$ of $h=160 \mathrm{~d}$ is larger than that of $h=75 \mathrm{~d}$, which may show that the bed of pebbles is higher and the area of the stagnant zone is larger. However, the area of the lower zone with the height $75 \mathrm{~d}$ in the pebble bed $h=160 \mathrm{~d}$ holds the great majority of the whole stagnant zone. It shows that the pebble flow in the lower zone plays a significant role in the formation of the stagnant zone.

(2) Moreover, the area of the lower zone with the height $75 \mathrm{~d}$ in the pebble bed $h=160 \mathrm{~d}$ is almost the same as the area of the stagnant zone of $h=75 \mathrm{~d}$. This fact indicates that the upper zone of the pebble bed has less impact on the mean-streamlines of the lower zone when the height is beyond a certain limit.

(3) The differences exist between the results of simulation and those of the experiment. The DEM may have its own disadvantages when calculating the area of the stagnant zone quantitatively, but it can be more useful for analyzing qualitatively.

Figure 9 shows the results about the distribution of the mean stagnant time of the experiments with $h=75 \mathrm{~d}$ and $h=$ $160 \mathrm{~d}$ with the same loading and discharging rate as above. The physical time in simulation was determined by means of counting the discharged pebbles.

In the experiment with $h=75 \mathrm{~d}$, the stagnant time of the marking pebble id 4 is more than ten times that of the marking pebble id 0 . The pebble id 4 is against the cylinder wall, and the

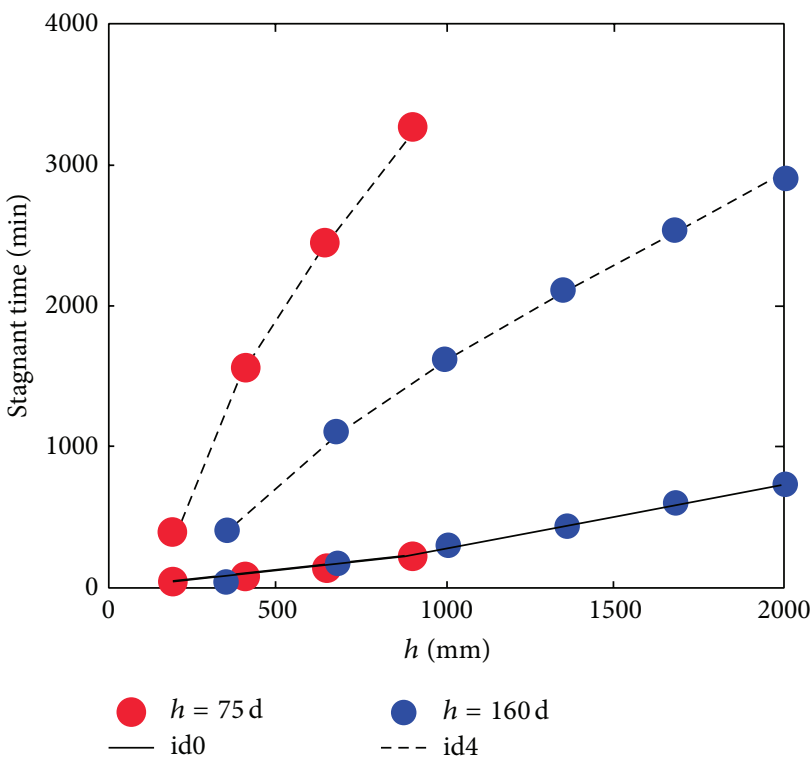

FIGURE 9: Experiment results of stagnant time of the id0, id4 and the stagnant time ratio id $2 / \mathrm{id} 0$, id $4 / \mathrm{id} 0$, of the experiment installation with $h=75 \mathrm{~d}$ and $h=160 \mathrm{~d}$.

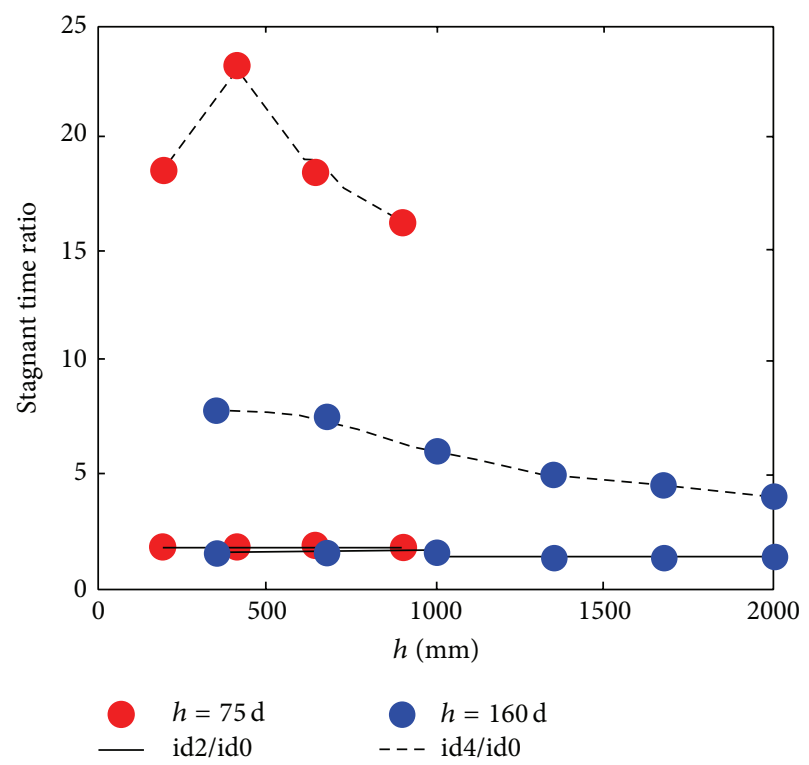

FIGURE 10: Experiment results of stagnant time ratio id2/id0, $\mathrm{id} 4 / \mathrm{id} 0$, of the experiment installation with $h=75 \mathrm{~d}$ and $h=160 \mathrm{~d}$.

id0 is in the central region, as shown in Figure 5. In order to explore the height's impact on stagnant time, Figures 9 and 10 show the experiment results of stagnant time of the id 0 , id 4 and the stagnant time ratio id $2 / \mathrm{id} 0$, id $4 / \mathrm{id} 0$, of the experiment installation with $h=75 \mathrm{~d}$ and $h=160 \mathrm{~d}$.

The experiment shows that the stagnant time ratio id $4 / \mathrm{id} 0$ of the experiment installation with $h=160 \mathrm{~d}$ is much smaller than that with $h=75 \mathrm{~d}$. The number of the ratio id1/id0, id2/id0, id3/id0 in the upper zone of the experiment with $h=$ $160 \mathrm{~d}$ is similar to that of the experiment with $h=1 \mathrm{~m}$ at the 

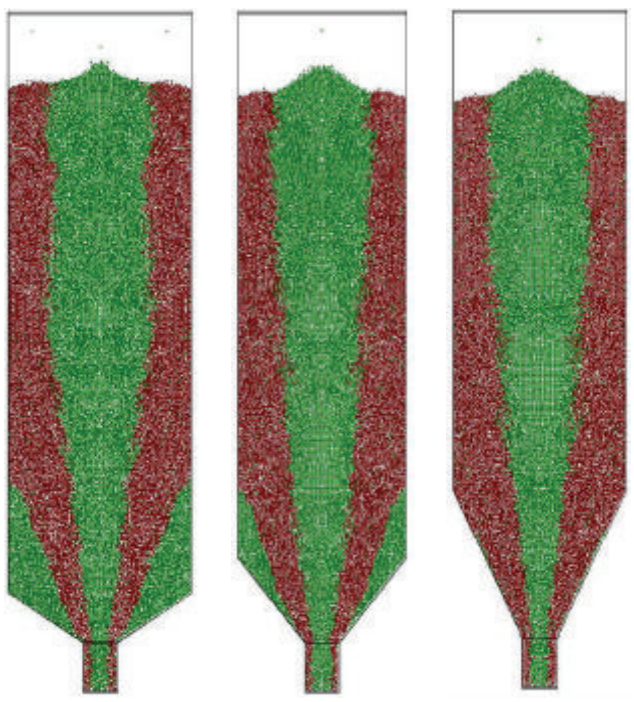

FIGURE 11: The result of the DEM simulation of the stagnant zone on the cone angles $30^{\circ}, 45^{\circ}$, and $60^{\circ}(f=0.2, h=160 \mathrm{~d})$.

same height. The pebble flow in the upper region has impact on the lower region, but the impact is not very large. However, the position of id4 against the cylinder wall is special. In the experiment with $h=75 \mathrm{~d}$, the id 4 is close to the cylinder wall, but in the experiment with $h=160 \mathrm{~d}$, at height $1 \mathrm{~m}$, the trace of id4 deviates from the cylinder wall. That is the reason why the ratio of stagnant time id $4 / \mathrm{id} 0$ of the experiment $h=75 \mathrm{~d}$ is much larger than that of the experiment with $h=160 \mathrm{~d}$. This is significant for us to find the way to avoid the stagnant zone.

3.3.2. Base Angle Effect. Figures 11 and 12 show the result of the DEM simulation of the stagnant zone on the cone angles $30^{\circ}, 45^{\circ}$, and $60^{\circ}$ under $f=0.2$ with pebble bed $h=160 \mathrm{~d}$.

We can find that the base cone angle is larger, and the stagnant zone is smaller, which plays a significant role in the flow field. When the base cone angle is not large enough, the slow zone exists. The pebbles in the stagnant zone are easier to move on a more inclined base slope, because the more inclined slope provides the larger driving force along the slope. That is why the increasing cone angle can decrease the scale of stagnant zone, and it almost disappears in the case of $60^{\circ}$ cone angle. In the case of $90^{\circ}$ cone angle, the whole pebbles are forced by the gravity and the friction with the wall, and the streamlines are almost vertical; then, the stagnant zone will disappear totally. So we can infer that the existence of the inclines of the wall plays a leading role in the forming of the stagnant zone.

Figures 13 and 14 indicate the simulation results about the mean stagnant time of the marking pebbles id0, id2, and id 4 and the stagnant time ratio of id2, id 4 to id0 in different height on the cone angles $30^{\circ}, 45^{\circ}$, and $60^{\circ}$ under $f=0.2$ on condition that the total height is $160 \mathrm{~d}$. The information can be inferred as follows.

(1) The stagnant time of the marking pebbles id 0 and id2 is almost the same at different heights on the angles

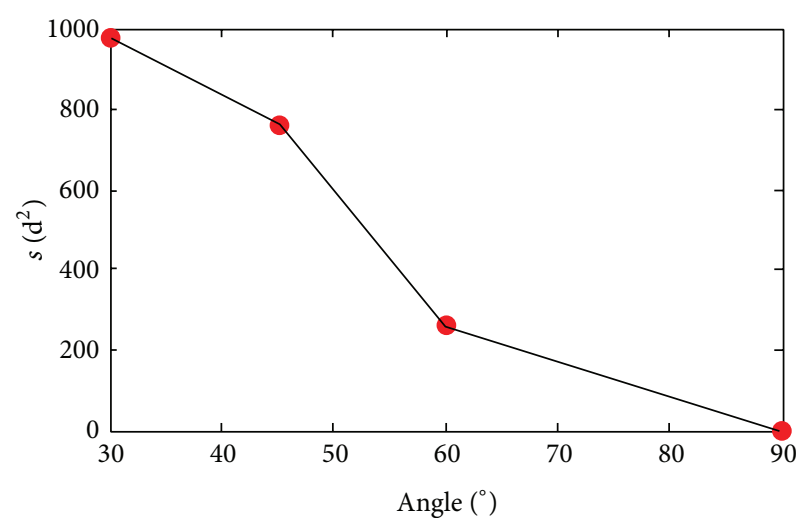

FIGURE 12: The simulation results about the cone angle and the stagnant zone size $(f=0.2, h=160 \mathrm{~d})$.

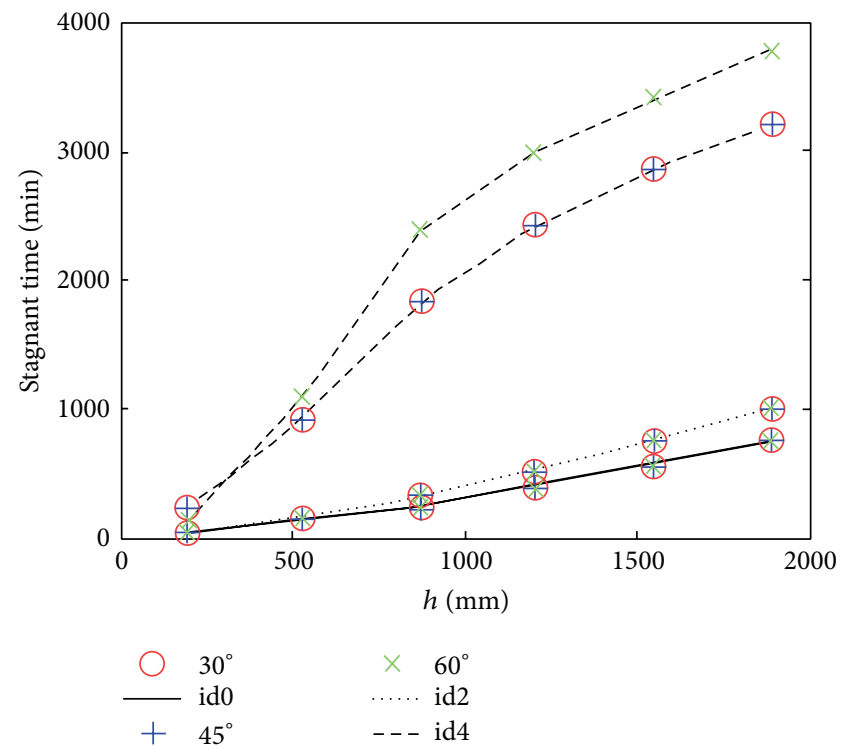

FIGURE 13: Simulation results about the mean stagnant time of the marking pebbles id $0, \mathrm{id} 2$, and id 4 , on the cone angles $30^{\circ}, 45^{\circ}$, and $60^{\circ}(f=0.2, h=160 \mathrm{~d})$.

$30^{\circ}, 45^{\circ}$, and $60^{\circ}$. Meanwhile, the pebbles id 0 and id 2 which are in the central zone of the pebble bed initially have passed the same vertical displacement, so we can infer that the changing of the cone angle has less impact on the size of the mean vertical velocity of the pebbles in the central zone with the same discharging and loading rate.

(2) The stagnant time of the pebble id 4 against the wall is much larger than that of id0 and id 2 in the central zone when the height of the pebble bed ranges from $200 \mathrm{~mm}$ to $1900 \mathrm{~mm}$ on different cone angles. Based on the consideration above, the existence of the inclines of the wall may be the main reason for the longer stagnant time of the pebbles against the wall.

(3) The stagnant time of id 4 does not change much on different cone angles. But the stagnant time of id 4 on 


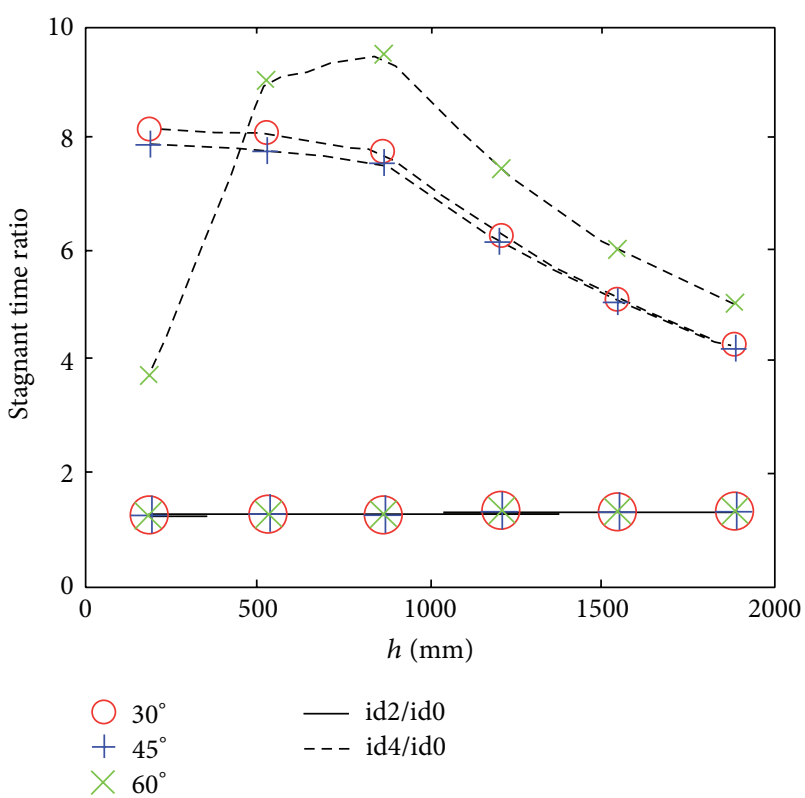

FIGURE 14: Simulation results about stagnant time ratio of id2, id 4 to id0 in different height on the cone angles $30^{\circ}, 45^{\circ}$, and $60^{\circ}(f=0.2$, $h=160 \mathrm{~d})$.

cone angle $60^{\circ}$ is larger than that on the angles $30^{\circ}$ and $45^{\circ}$, while the stagnant time of id 4 on angles $30^{\circ}$ and $45^{\circ}$ is almost the same. As we can see in Figure 13, the pebble id4 transferring from $h=1000 \mathrm{~mm}$ to $h=500 \mathrm{~mm}$ takes up longer time than the other two angles. It may relate with the fact that the meanstreamline of id 4 on $60^{\circ}$ is much closer to the wall than the other two cone angles. This fact must be paid attention to while designing the reactor core.

3.3.3. Friction Coefficient Effect. The friction coefficient offers more complicated influence on pebble flow and presents a strong nonlinearity. The related work was yet very insufficient. The simulation results of the stagnant zone shape and size have been shown in Figure 15 under $f=0.1, f=0.2$, and $f=0.4$ on the base cone angle $30^{\circ}$ with $h=75 \mathrm{~d}$.

As is shown in Figure 16, when the friction coefficient is small enough, for example, $f=0.1$, the stagnant zone decreases but has not yet disappeared due to being far away from the drainage hole. However, this kind of change of the size of stagnant zone is not linear.

The driving force and resistance force influence the existence, shape, and size of the stagnant zone. The stagnant zone receives not only the resistance from the base wall but also the driving force from the outer flowing pebbles. When friction coefficient increases from 0.1 to 0.2 , the stagnant zone tends to increase by a large margin because the resistance force between the stagnant zone and the base wall plays a more important role than the driving force between stagnant zone and the outer flowing pebbles. Meanwhile, when the friction coefficient becomes from 0.2 to 0.4 , the stagnant zone tends not to increase obviously due to the increase of the driving force from outer neighbor pebbles.
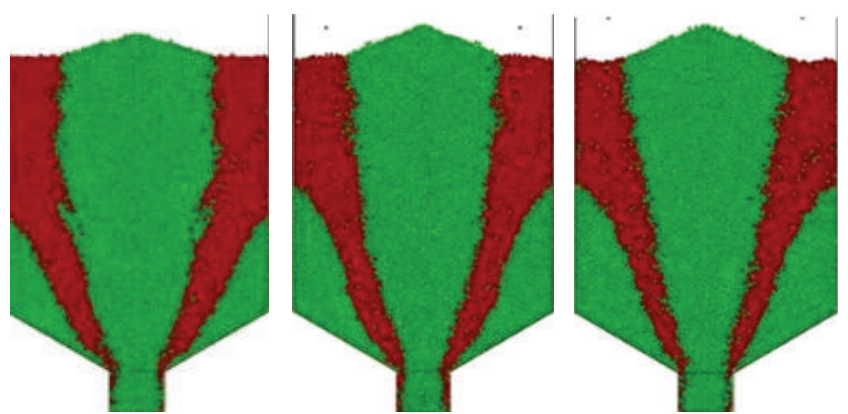

FIGURE 15: The result of the DEM simulation of the stagnant zone on the friction coefficients $0.1,0.2$, and $0.4\left(A=30^{\circ}, h=160 \mathrm{~d}\right)$.

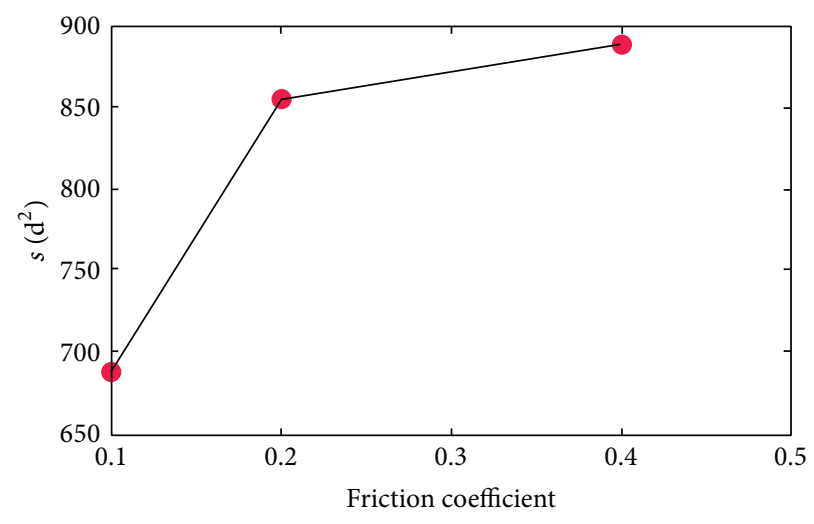

FIgURE 16: The simulation results about the friction coefficient and the stagnant zone size $(f=0.2, h=160 \mathrm{~d})$.

\section{Conclusion}

In this paper, the stagnant zone in the gravity-driven pebble bed has been analyzed via the experimental method (side area method) and DEM simulation. The mean-streamline method to define stagnant zone and to calculate the proportion of this has been proposed with considering the undesirable effect of the mixing zone. Meanwhile, the stagnant time method with tracking the time of the marking balls until they are discharged from the orifice has been applied to explore the kinematics characteristics of the pebbles with different coordination in the quasi-static pebble flow field. We also discussed the effects of bed configuration on stagnant zone including the bed height effect, base angle effect, and the friction coefficient effect and can summarize as follows.

(1) The pebble flow in the lower zone plays a significant role in the formation of the stagnant zone. The upper zone of the pebble bed has less impact on the meanstreamlines of the lower zone when the height is beyond a certain limit. Bed height affects the stagnant zone slightly when exceeding a certain height.

(2) The base angle has negative prominent correlation with the stagnant zone; the angle is larger, and the area of the stagnant zone is smaller. The existence of the inclines of the wall plays a leading role in the formation of the stagnant zone. 
(3) The area of the stagnant zone increases more rapidly with the friction coefficient from 0.1 to 0.2 than that with the friction coefficient from 0.2 to 0.4 . The mechanism about how the friction influences the area of the stagnant zone and the overall flow field deserves to be deeply investigated.

(4) Thus, it could be suggested that smooth transition from bases to sides, larger base cone angle or discharge hole, and less width of the vessel will be favorable to avoid the stagnant region.

\section{Disclosure}

This paper has been approved by all authors; none of the material presented in the paper is submitted or published elsewhere; and the paper does not contain any information with restricted access or proprietary content.

\section{Conflict of Interests}

The authors declare that there is no conflict of interests regarding the publication of this paper.

\section{Acknowledgments}

The authors are grateful for the support of this research by the National Natural Science Foundation of China (Grants nos. 11072131 and 51106180) and China Postdoctoral Science Foundation (2013M540964).

\section{References}

[1] W. Magwood, "Roadmap to the next generation nuclear power systems: a vision for a powerful future," Nuclear News, vol. 43, pp. 35-37, 2000.

[2] Z. Y. Zhang, Z. X. Wu, Y. H. Xu, Y. L. Sun, and F. Li, "Design of Chinese modular high-temperature gas-cooled reactor HTRPM," in Proceedings of the 2nd International Topical Meeting on High Temperature Reactor Rechnology, Beijing, China, Septrmber 2004.

[3] A. Koster, H. D. Matzner, and D. R. Nicholsi, "PBMR design for the future," Nuclear Engineering and Design, vol. 222, no. 2-3, pp. 231-245, 2003.

[4] A. C. Kadak and M. V. Berte, "Modularity in design of the MIT pebble bed reactor," in Proceedings of the American Nuclear Society Meeting, Reno, Nev, USA, November 2001.

[5] R. Schulten, "Pebble bed HTRs," Annals of Nuclear Energy, vol. 5, no. 8-10, pp. 357-374, 1978.

[6] H. M. Jaeger, S. R. Naegel, and R. P. Behringer, "Granular solids, liquids and gases," Reviews of Modern Physics, vol. 68, no. 4, pp. 1259-1273, 1996.

[7] J. M. Ottino and D. V. Khakhar, "Mixing and segregation of granular materials," Annual Review of Fluid Mechanics, vol. 32, pp. $55-91,2000$.

[8] D. Talbot, “The next nuclear plant," Technology Review, vol. 105, no. 1, pp. 54-59, 2002.

[9] J. Choi, A. Kudrolli, and M. Z. Bazant, "Velocity profile of granular flows inside silos and hoppers," Journal of Physics Condensed Matter, vol. 17, no. 24, pp. S2533-S2548, 2005.
[10] S. B. Rochal, V.P. Dmitriev, V .L. Lorman, and P. Tolédano, "Local mechanism for crystal-quasicrystal transformations," Physics Letters A, vol. 220, no. 1-3, pp. 111-116, 1996.

[11] A. Samadani, A. Pradhan, and A. Kudrolli, "Size segregation of granular matter in silo discharges," Physical Review E, vol. 60, no. 6, pp. 7203-7209, 1999.

[12] J. Choi, A. Kudrolli, R. R. Rosales, and M. Z. Bazant, "Diffusion and mixing in gravity-driven dense granular flows," Physical Review Letters, vol. 92, no. 17, Article ID 174301, 2004.

[13] A. Medina, J. Andrade, and C. Treviño, "Experimental study of the tracer in the granular flow in a $2 \mathrm{D}$ silo," Physics Letters A, vol. 249, no. 1-2, pp. 63-68, 1998.

[14] D. M. Mueth, G. F. Debregeas, G. S. Karczmar, P. J. Eng, S. R. Nagel, and H. M. Jaeger, "Signatures of granular microstructure in dense shear flows," Nature, vol. 406, no. 6794, pp. 385-389, 2000.

[15] E. R. Weeks, J. C. Crocker, A. C. Levitt, A. Schofield, and D. A. Weitz, "Three-dimensional direct imaging of structural relaxation near the colloidal glass transition," Science, vol. 287, no. 5453, pp. 627-631, 2000.

[16] J.-C. Tsai and J. P. Gollub, "Slowly sheared dense granular flows: crystallization and nonunique final states," Physical Review E, vol. 70, no. 3, Article ID 031303, 2004.

[17] M. Menon and D. J. Durian, "Diffusing-wave spectroscopy of dynamics in a three-dimensional granular flow," Science, vol. 275, no. 5308, pp. 1920-1922.

[18] J. S. Goodling, R. I. Vachon, W. S. Stelpflug, and S. J. Ying, "Radial porosity distribution in cylindrical beds packed with spheres," Powder Technology, vol. 35, no. 1, pp. 23-29, 1983.

[19] Y. Cohen and A. B. Metzner, "Wall effects in laminar flow of fluids through packed beds," AIChE Journal, vol. 27, no. 5, pp. 705-715, 1981.

[20] R. M. Nedderman and U. Tüzün, "A kinematic model for the flow of granular materials," Powder Technology, vol. 22, no. 2, pp. 243-253, 1979.

[21] W. W. Mullins, "Stochastic theory of particle flow under gravity," Journal of Applied Physics, vol. 43, no. 2, pp. 665-678, 1972.

[22] M. Z. Bazant, "The Spot model for random-packing dynamics," Mechanics of Materials, vol. 38, no. 8-10, pp. 717-731, 2006.

[23] P. A. Cundall and O. D. L. Strack, "A discrete numerical model for granular assemblies," Géotechnique, vol. 29, no. 1, pp. 47-65, 1979.

[24] X. T. Yang, W. P. Hu, S. Y. Jiang, K. K. L. Wong, and J. Y. $\mathrm{Tu}$, "Mechanism analysis of quasi-static dense pebble flow in pebble bed reactor using phenomenological approach," Nuclear Engineering and Design, vol. 250, pp. 247-259, 2012.

[25] C. H. Rycroft, G. S. Grest, J. W. Landry, and M. Z. Bazant, "Analysis of granular flow in a pebble-bed nuclear reactor," Physical Review E, vol. 74, no. 2, Article ID 021306, 2006. 


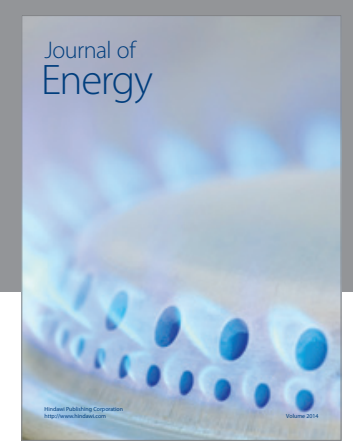

Journal of

Industrial Engineering
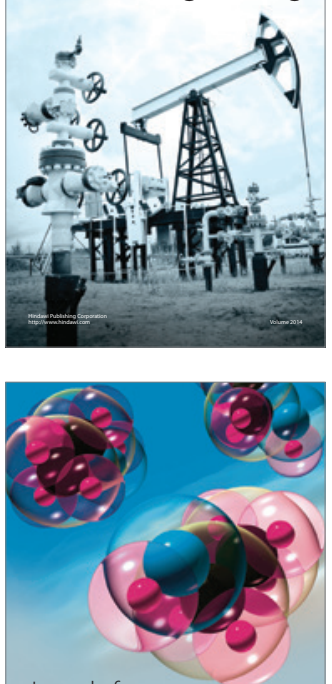

Fuels
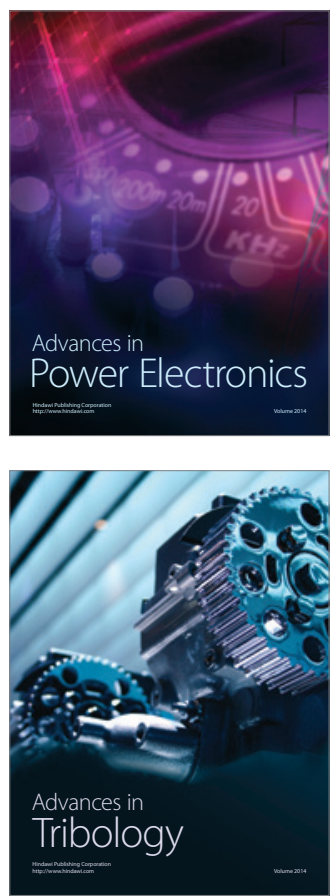

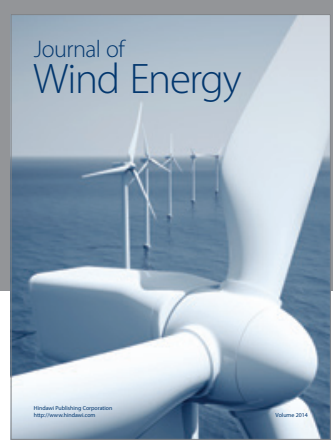

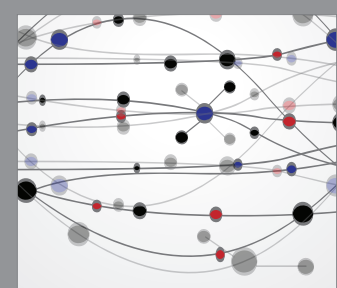

The Scientific World Journal

Submit your manuscripts at http://www.hindawi.com

Journal of

Structures
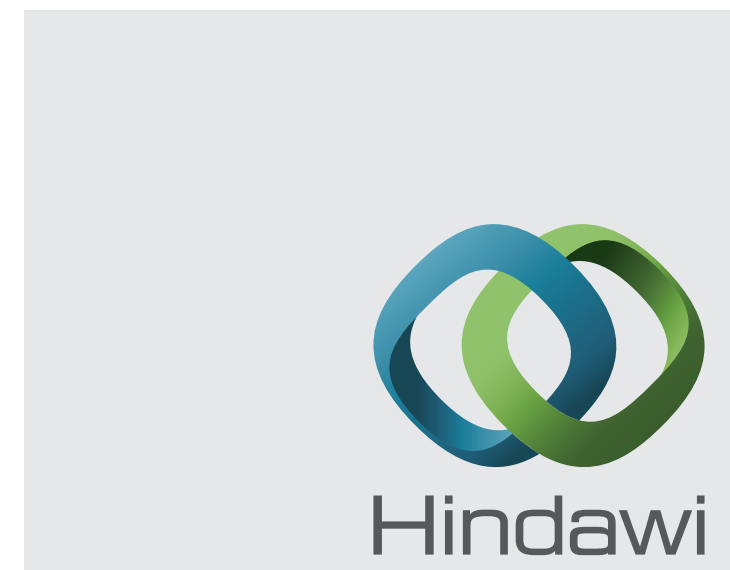

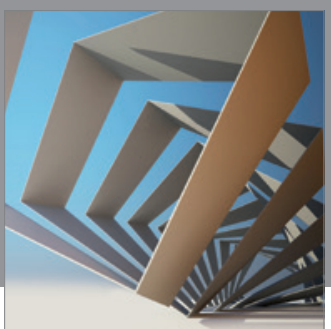

Rotating

Machinery
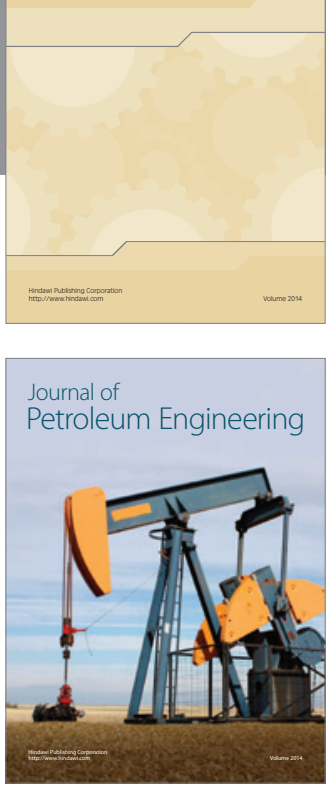

Journal of

Solar Energy
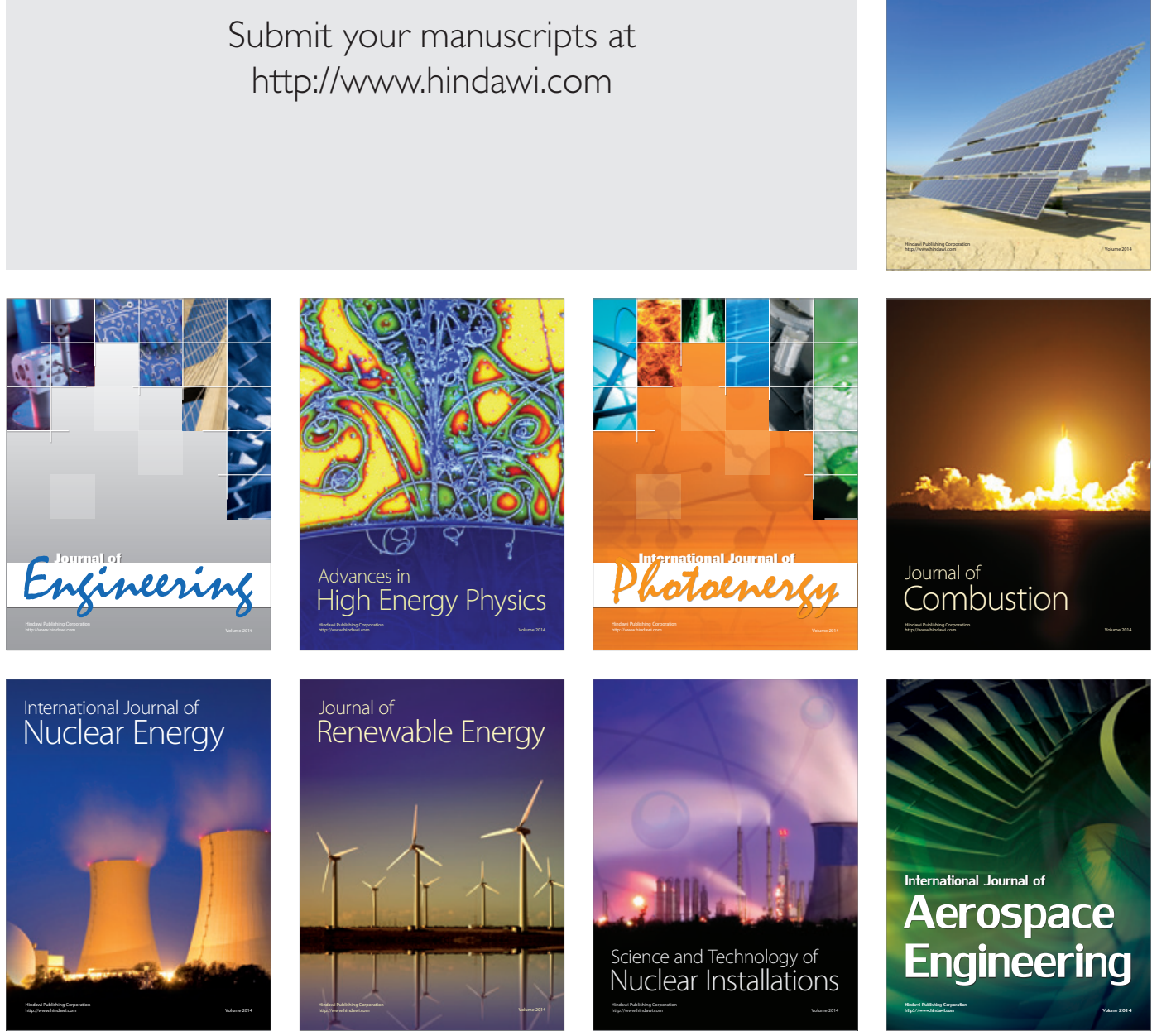